\title{
Approach to novel functional foods for stress control 2. Microarray assessment of exercise in healthy volunteers
}

\author{
Yutaka Nakaya', Yasuko Hata', Kaori Ishida', Akira Takahashi', Kyoko Morita², and \\ Kazuhito Rokutan ${ }^{2}$ \\ ${ }^{1}$ Departments of Nutrition and Metabolism, and ${ }^{2}$ Stress science, Institute of Health Biosciences, The \\ University of Tokushima Graduate School, Tokushima, Japan
}

\begin{abstract}
DNA microarray was used to measure stress response by exercise in peripheral blood leukocytes. Aerobic exercise did not alter mRNA pattern or urinary secretion of 8-hydroxy-2'deoxyguanosine (8-OHdG) . Strenuous exercise increased urinary secretion of 8-OHdG and altered mRNA pattern in microarray. These results suggest that moderate exercise, i. e. aerobic exercise, did not show any change in 8-OHdG, an oxidative stress marker, or mRNA expression in the leukocytes, which might reflect whole body neurohormanal changes. In addition, strenuous exercise produced quite different expression pattern from those of psychological stress. J. Med. Invest. 52 Suppl. : 242-243, November, 2005
\end{abstract}

Keywords : DNA microarray, exercise, aerobic exercise, anaerobic exercise

\section{OBJECTIVE}

DNA microarray technology has greatly enhanced research exploring transcriptional contributions to cellular events. To clarify the characteristics of two types of stress, i. e. physical stress and mental stress, we compared mRNA expression after exercise and psychological stress after examination.

\section{METHODS}

We studied a microarray including 1467 cDNAs developed by Rokutan $\mathrm{K}^{1}$ that were selected to specifically measure stress response in peripheral blood leukocytes. The mRNA levels in leukocytes were compared with those before and 4, 24 and 48 hours after exercise by bicycle ergometer. Urinary

Received for publication September 9, 2005 ; accepted September 16, 2005.

Address correspondence and reprint requests to Yutaka Nakaya, M. D., Ph. D., Department of Nutrition and Metabolism, Institute of Health Biosciences, The University of Tokushima Graduate School, Kuramoto-cho, Tokushima 770-8503, Japan and Fax : + 81-88-633-7113. secretion of 8-hydroxy-2'-deoxyguanosine (8-OHdG) were measured and normalized by urea nitrogen. Two types of exercise were carried out, 1) aerobic exercise $\left(50-60 \%\right.$ of maximal $\left.\mathrm{VO}_{2}\right)$ for one hour $(3 \times 20 \mathrm{~min}$ exercise and $5 \mathrm{~min}$ rest $)$ and 2$)$ strenuous exercise until exhausted (25 Watt increment of load every 5 min until exhausted). In psychological stress, venous blood was collected from 10 graduate students $2 \mathrm{~h}$ before and 2 or $24 \mathrm{~h}$ after an open presentation for their $\mathrm{Ph}$. D. The mRNA levels in leukocytes were compared with those prepared 4 weeks before the presentation $(1,2)$.

\section{RESULTS AND DISCUSSION}

Aerobic exercise did not alter mRNA pattern or urinary secretion of 8-OHdG (an indicator for oxidative stress). Strenuous exercise up to exhaustion produced significant elevation of urinary 8-OHdG. DNA microarray study showed that 17 mRNA were increased $(>2$ times) or decreased $(<1 / 2)$, which number was much smaller than those in open presentation for PH. D students, i. e. up-regulated 49 genes and down-regulated 21 genes $(1,2)$. In addition, the degrees of increased 
mRNA levels were not correlated to the change in 8-HDG in strenuous exericise. Interestingly, these mRNA were quite different from those in open presentation of the $\mathrm{Ph}$. D students, or chronic fatigue syndrome (data not shown). The altered expressions after strenuous exercise were mainly in mRNA of oxidant stress response and proteolysis.

These results suggest that moderate exercise, i. e. aerobic exercise, did not show any change in stress markers or mRNA expression in the leukocytes, which might reflect whole body neurohormanal changes. However, even strenuous (anaerobic) stress for up to $15 \mathrm{~min}$, the change in the pattern of RNA is much smaller in numbers compared to those of psychological stress when we use this microarray system.

\section{CONCLUSION}

In this study we found psychological stress produced larger change in mRNA pattern in leukocytes compared to strenuous exercise. We did not analyze microarray study in working muscle in the present study. However, the results suggest that the hypothalamus-pituitaryadrenal (HPA) axis, sympathetic nervous system, and immune system, which affect the mRNA expression pattern of leukocytes, are more affected by psychological stress than by physical stress.

\section{REFERENCES}

1. Rokutan K, Morita K, Masuda K, Tominaga K, Shikishima M, Teshima-Kondo S, Omori T, Sekiyama A: Gene expression profiling in peripheral blood leukocytes as a new approach for assessment of human stress response. J Med Invest 52 : 137-44, 2005

2. Morita K, Saito T, Ohta M, Ohmori T, Kawai K, Teshima-Kondo S, Rokutan K: Expression analysis of psychological stress-associated genes in peripheral blood leukocytes. Neurosci Lett 381: $57-62,2005$ 